\title{
ANALYSIS OF A PRACTICAL QUAD COPTER ROBOT USING LINEAR QUADRATIC REGULATOR CONTROLLER
}

\author{
Ahmed Y. Abo Taleb, Hala S. El-sayed, H. A. Yassin \\ Department of Electrical Engineering, Menoufia University, Shebin El-kom, Egypt
}

\begin{abstract}
This paper aims to evaluate a practical implementation of an efficient control system for a quad copter robot under Linear Quadratic Regulator. Simulation results are conducted using Matlab\Simulink. Experimental results with different values of Euler angles demonstrated that the Linear Quadratic Regulator controller provide a robust, versatile and easy implementable controller system.
\end{abstract}

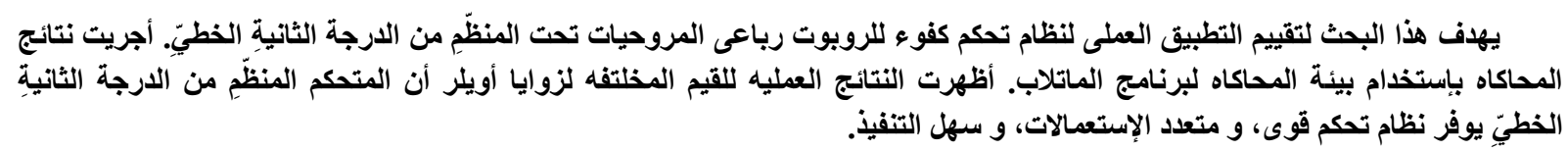

Keywords : Quad-rotor helicopter, Linear Quadratic Regulator (LQR) controller, Matlab Simulink

\section{INTRODUCTION}

Unmanned Aerial Vehicles (UAVs) are very versatile aircrafts in contrast to their lower complexity; it can be called the robot future due to their various advantages in our life rather than conventional aircrafts [1]. In the past decade, there has been growing interest in the use of UAVs for both government and commercial applications [2, 3]. As UAV technology improves, unmanned vehicles are playing a greater role in national military operations with missions ranging from early warning and maritime tracking to communications relay [4, 5]. Quad copter robots are a particular type of UAVs, which also may be referred as a quad copter helicopter. Within UAV hardware, quad copters are being widely used for different purposes, such as educational, commercial or entertainment. The first type of quad copter was flown in the 1920's to be used as manned vehicles, but the first successful manned flight was in the1960's when the CurtissWright X-19A was developed. However, these early quad copters lacked stability and controlling during flight. With recent advances in on board computer technology, quad copter stability and control is much easier. Therefore, In order to perform an efficient stabilizing system and navigation, there are several techniques implemented for controlling quad copters robot, each one with its peculiarities $[6,7]$.This work design and apply LQR control technique for a quad copter robot having a tri- axis accelerometer and a compass as the sensors. Researches consider the LQR controller is a good controller because of its great performance and robustness in the plant in question [8, 9, and 10]. This paper is organized as follows: Section 2 describes the operation and experimental framework of a quad copter robot. Section 3 explores the dynamic modeling of a quad copter robot. Section 4 explains the LQR technique applied for a quad copter robot. Section 5 presents simulation and experiment angular velocities and Euler angles of the quad copter robot provided by the available sensors. Finally, Section 6 introduces conclusions.

\section{OPERATION OF A QUAD COPTER ROBOT}

The quad copter robot is consists of 4 arms arranged on $\mathrm{x}$ shape or + shape, every arm holds a propeller with a brushless dc motor on its end (28 A, 12 V DC, Max thrust: $1300 \mathrm{~g}$ ). As shown in figure1, the configuration presents a very low moment of inertia and six degrees of freedom (three translational and three rotational), which results in great stability of the quad copter.

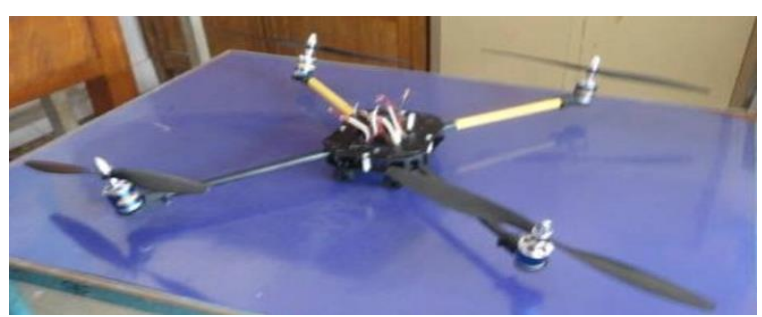

Figure1 Experimental framework of a quad copter robot 
Conventional helicopters have two rotors arranged as two coplanar rotors both providing upwards thrust, but spinning in opposite directions in order to balance the torques exerted upon the body of the helicopter. However, these configurations require complicated machinery to control the direction of motion. With new versions of a quad copter, the operation is based on four rotors. Each rotor is responsible for a certain amount of thrust and torque about its center of the rotation, as well as for a drag force opposite to the rotorcraft's direction of flight. In fact, the quad copter's propellers are not similar; they are divided in two pairs, two pushers and two puller blades that work in contra-rotation. As a consequence, the resulting net torque can be null if all propellers turn with the same angular velocity and the aircraft still around its center of gravity. In order to define an aircraft's movement around its center of mass that describe all possible combinations of aircraft attitude, aerospace engineers usually define three rotational parameters, the angles of yaw, pitch and roll. It is useful because of the forces used to control the aircraft act around its center of mass, causing it to pitch, roll and yaw. As indicated in figure 2, changes in the pitch angle are induced by contrary variation of speeds in propellers 1 and 3, resulting in forward or backwards translation. With the same action for propellers 2 and 4 , can produce a change in the roll angle and will get lateral translation. Yaw is induced by mismatching the balance in aerodynamic torques.

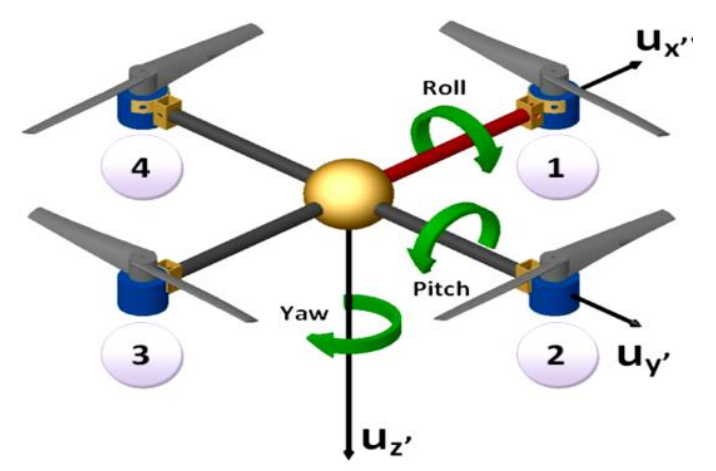

Figure 2: Yaw, pitch and roll rotations

\section{QUAD COPTER MODELING}

For describing the quad copter attitudes, the equations and the parameters needed to generate for each element;

\subsection{System Dynamics}

Due to the presence of two coordinate systems, it is necessary to use the transformation matrix to obtain the response of any movement from a coordinate system (Earth-fixed frame) to the other (model-fixed frame or mobile frame). It will designate this reference frame by North-East-Down $\left(\mathrm{O}_{\mathrm{NED}}\right)$, because two of its axis $\left(\mathrm{u}_{\mathrm{x}}\right.$ and $\left.\mathrm{u}_{\mathrm{y}}\right)$ are aligned respectively with the North and East direction, and the third axis $\left(\mathrm{u}_{\mathrm{z}}\right)$ is directed down, aligned towards the center of the Earth (Figure .2). The mobile frame or aircraft body is designated by $\mathrm{O}_{\mathrm{ABC}}$ and its origin coincident with the quad copter's center of gravity.

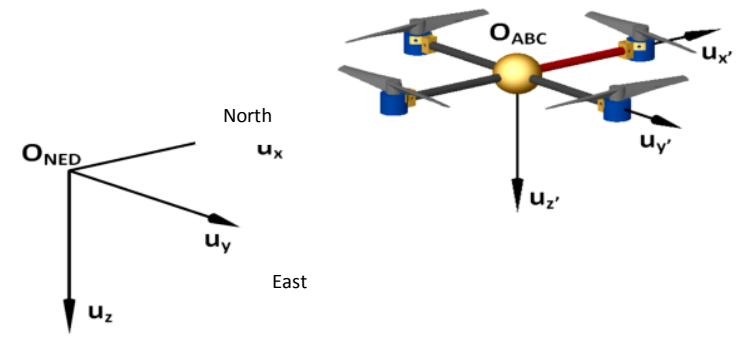

Figure 3: NED and ABC reference frames.

In control theory, the dynamic behavior of a given system can be understood through its states. For a quad copter, the dynamic behavior about 3 axis of rotation is examined first with 6 states: the Euler angles $[\varphi, \theta, \psi]$ (Roll - Pitch - Yaw) and the angular velocities around each axis of the $\mathrm{O}_{\mathrm{ABC}}$ frame $[P Q$ $R]$. Second, another 6 states are necessary: the position of the Center of Gravity (COG) $[X Y Z]$ and respective linear velocity components $\left[\begin{array}{lll}U & V & W\end{array}\right]$ relative to the fixed frame. Therefore, the quad copter has 12 states that describe 6 degrees of freedom. The equations describing the orientation of the mobile frame relative to the fixed one can be deduced by using a rotation matrix $\mathrm{S}$. This matrix results of the product between three matrices $(R(\varphi), R(\theta)$ and $R$ $(\psi)$ ), each of them representing the rotation of the $\mathrm{ABC}$ frame around the $\mathrm{O}_{\mathrm{NED}}$ axis

$$
S=\left[\begin{array}{ccc}
c \theta c \psi & c \theta s \psi & -s \theta \\
s \psi s \theta c \psi-c \varphi s \psi & c \varphi c \psi+s \varphi s \theta s \psi & s \varphi c \theta \\
c \varphi s \theta c \psi+s \varphi s \psi & s \theta c \varphi s \psi-s \varphi c \psi & c \theta c \varphi
\end{array}\right]
$$

Where; $s=\sin , c=\cos$.

The vectors of the linear accelerations acting on the vehicle's body are: 


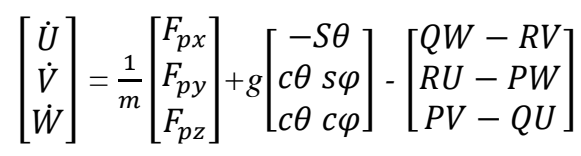

Where; $\left[F_{P x} F_{P y} F_{P z}\right]$ are the vector elements of $F_{P}$.

Assuming the aircraft is in a hovered flight, in such a scenario, the forces are acting only in the $\mathrm{z}$ axis of quad copter, corresponding to the situation where it have the engines trying to overcome the force of gravity to keep the aircraft stable at a given altitude:

$$
F_{p z}=-\left(T_{1}+T_{2}+T_{3}+T_{4}\right)
$$

Assuming the quad copter is a rigid body with constant mass and axis aligned with the principal axis of inertia and then, the tensor $\boldsymbol{I}$ becomes a diagonal matrix containing only the principal moments of inertia:

$$
\boldsymbol{I}=\left[\begin{array}{ccc}
I_{11} & 0 & 0 \\
0 & I_{22} & 0 \\
0 & 0 & I_{33}
\end{array}\right]
$$

Therefore, The vector of angular accelerations:

$$
\left[\begin{array}{l}
\dot{\mathrm{P}} \\
\dot{Q} \\
\dot{\mathrm{R}}
\end{array}\right]=\left[\begin{array}{l}
\frac{M_{x}}{I_{11}} \\
\frac{M_{y}}{I_{22}} \\
\frac{M_{Z}}{I_{33}}
\end{array}\right]-\left[\begin{array}{l}
\frac{\left(I_{33}-I_{22}\right) Q R}{I_{11}} \\
\frac{\left(I_{11}-I_{33}\right) R P}{I_{22}} \\
\frac{\left(I_{22}-I_{11}\right) P Q}{I_{33}}
\end{array}\right]
$$

The moments acting on the aircraft can be provided by:

$$
\begin{aligned}
M_{x}=\left(T_{4}-T_{2}\right) d c g & (6) \\
M_{y}=\left(T_{1}-T_{3}\right) d c g & (7) \\
M_{z}= & \left(T_{1}+T_{3}-T_{2}-T_{4}\right) K_{T M}
\end{aligned}
$$

Where $d_{c g}$ is the distance to the aircrafts COG and $K_{T M}$ is a constant that relates moment and thrust of a propeller.

\subsection{Kinematic Equations and Euler Angles}

The flight path of the quad copter in terms of $[X$ $Y Z]$ can be found by integration of equation

$$
\left[\begin{array}{c}
\dot{\mathrm{X}} \\
\dot{\mathrm{Y}} \\
\dot{\mathrm{z}}
\end{array}\right]=S^{T}\left[\begin{array}{c}
U \\
V \\
W
\end{array}\right]
$$

The rate of change of the Euler angles:

$$
\left[\begin{array}{c}
\dot{\varphi} \\
\dot{\theta} \\
\dot{\psi}
\end{array}\right]=\left[\begin{array}{ccc}
1 & \tan \theta \sin \varphi & \tan \theta \cos \varphi \\
0 & \cos \varphi & -\sin \varphi \\
0 & \sin \varphi / \cos \theta & \cos \varphi / \cos \theta
\end{array}\right]\left[\begin{array}{l}
P \\
Q \\
R
\end{array}\right]
$$

\subsection{Quaternion Differential Equations}

If the aircraft pitches up 90 degrees, the aircraft roll axis becomes parallel to the yaw axis, and there is no axis available to accommodate yaw rotation (one degree of freedom is lost). To overcome this problem, the quaternion method may be used. The quaternion vector elements as a function of Euler angles yields:

$\left[\begin{array}{l}q_{0} \\ q_{1} \\ q_{2} \\ q_{3}\end{array}\right]=\left[\begin{array}{l}\cos \left(\frac{\psi}{2}\right) \cos \left(\frac{\theta}{2}\right) \cos \left(\frac{\varphi}{2}\right)+\sin \left(\frac{\psi}{2}\right) \sin \left(\frac{\theta}{2}\right) \sin \left(\frac{\varphi}{2}\right) \\ \cos \left(\frac{\psi}{2}\right) \cos \left(\frac{\theta}{2}\right) \cos \left(\frac{\varphi}{2}\right)-\sin \left(\frac{\psi}{2}\right) \sin \left(\frac{\theta}{2}\right) \sin \left(\frac{\varphi}{2}\right) \\ \cos \left(\frac{\psi}{2}\right) \sin \left(\frac{\theta}{2}\right) \cos \left(\frac{\varphi}{2}\right)+\sin \left(\frac{\psi}{2}\right) \cos \left(\frac{\theta}{2}\right) \sin \left(\frac{\varphi}{2}\right) \\ \sin \left(\frac{\psi}{2}\right) \cos \left(\frac{\theta}{2}\right) \cos \left(\frac{\varphi}{2}\right)-\cos \left(\frac{\psi}{2}\right) \sin \left(\frac{\theta}{2}\right) \sin \left(\frac{\varphi}{2}\right)\end{array}\right]$

Also gets the absolute velocity using the quaternion rotation tensor:

$$
\left[\begin{array}{c}
\dot{\mathrm{X}} \\
\dot{\mathrm{Y}} \\
\dot{\mathrm{Z}}
\end{array}\right]=S_{q}^{T}\left[\begin{array}{c}
U \\
V \\
W
\end{array}\right]
$$

To employ the quaternion method combines the previous quaternion equations with the dynamics equations to compose the vehicle acceleration on the aircraft local frame:

$$
\left[\begin{array}{c}
\dot{U} \\
\dot{V} \\
\dot{W}
\end{array}\right]=\frac{1}{m}\left[\begin{array}{c}
F_{p x} \\
F_{p y} \\
F_{p z}
\end{array}\right]+g\left[\begin{array}{c}
2\left(q_{1} q_{3}-q_{0} q_{2}\right) \\
2\left(q_{2} q_{3}+q_{0} q_{1}\right) \\
q_{0}^{2}-q_{1}^{2}-q_{2}^{2}+q_{3}^{2}
\end{array}\right]-\left[\begin{array}{c}
Q W-R V \\
R U-P W \\
P V-Q U
\end{array}\right]
$$

\subsection{Sensors Modeling}

\section{A) Accelerometer}

Absolute acceleration of the accelerometer located at any point $p$ :

$$
a_{P}=\frac{a_{B}}{m}+S\left[\begin{array}{lll}
0 & 0 & g
\end{array}\right]^{T} \dot{\omega}_{B} \times r_{S}+\omega_{B} \times\left(\omega_{B} \times r_{S}\right)
$$

Where; $r_{s}$ is the position of the accelerometer relative to the quad copter's center of gravity, $a_{p}$ is the absolute acceleration at point $\mathrm{p}$. To obtain these 
angles through the vector of gravitational acceleration provided by the accelerometer are:

$$
\begin{aligned}
& \phi=\arctan \left(\frac{a_{p y}}{a_{p z}}\right) \\
& \theta=-\arctan \left(\frac{a_{p x}}{a_{p z}}\right)
\end{aligned}
$$

\section{B) Compass}

It can be modeled as follows:

$$
\psi=N
$$

Where, $N$ is the direction of the magnetic North Magnetic Pole mapped between $-\pi$ and $\pi$ radians.

\section{QUAD COPTER CONTROL}

\subsection{Kalman Filter}

The dynamic model of the quad copter is not linear which causes noisy under control. The Kalman filter [8] is a recursive filter created for a linear system to estimate states error tries to go to zero while under the influence of noise (e.g. sensor readings).Linearizing the system will facilitate the construction of the Kalman filter and quad copter control. Let us take as a starting point for the process of linearization of the state vector $\bar{x}$, which illustrates nothing less than the quad copter in flight, stabilized at a height $Z$ from the ground. This point is usually known as an equilibrium point. The linearization of the quad copter's model:

$$
\begin{aligned}
& \bar{x}=\left[\begin{array}{llllllllllll}
U & V & W & P & Q & R & X & Y & Z & \phi & \theta & \psi
\end{array}\right]^{T}= \\
& {\left[\begin{array}{llllllllllll}
0 & 0 & 0 & 0 & 0 & 0 & 0 & 0 & -z & 0 & 0 & 0
\end{array}\right]^{T}}
\end{aligned}
$$

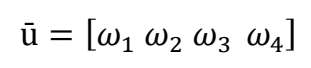

$$
\begin{aligned}
& \dot{\mathrm{x}}=f(x, u) \quad y=h(x, u)(20)
\end{aligned}
$$

Where; $u$ is the speed of rotation vector of each one of the motors, $x$ is the state vector. The linearized state space representation around the hovering conditions:

$$
\begin{aligned}
& \dot{\mathrm{x}}=A x+B u \\
& y=C x+D u
\end{aligned}
$$

With:

$$
\begin{aligned}
& A=\frac{\partial f(x, u)}{\partial x} \\
& B=\frac{\partial f(x, u)}{\partial u} \\
& C=\frac{\partial h(x, u)}{\partial x} \\
& D=\frac{\partial h(x, u)}{\partial u}
\end{aligned}
$$

\subsection{LQR Controller}

In optimal control one endeavor on finding a controller that provides the best possible performance with respect to some given measure of performance. In 2005, Castillo et al. has implemented LQR controller in which the control signal energy is measured by a cost function containing weighting factors provided by the controller designer [12]. During simulation the controller has performed satisfactory. For a continuous-time linear system described by the equation (21). The cost function $J_{L Q R}$ is:

$$
\begin{gathered}
J_{L Q R}=\int_{0}^{\infty}\left(x^{T} \hat{Q} x+u^{T} \hat{R} u\right) d t \\
u^{K}=-\bar{K} x
\end{gathered}
$$

Where $Q$ is a square matrix of sixth order, $\hat{R}$ is a unitary vector and $u^{K}$ is the vector of control actions. The LQR gain matrix $\bar{K}$ is provided by:

$$
\bar{K}=\hat{R}^{-1} B^{T} \hat{P}
$$

$P$ is derived by means of the algebraic Riccati equation:

$$
A^{T} \hat{P}+\hat{P} A-\hat{P} B \hat{R}^{-1} B^{T} \hat{P}+\hat{Q}=0
$$

\section{IMPLEMENTATION AND RESULTS}

Hardware implementation of the quad copter in the Arduino chip is quite easy as indicated in figure 4. The mathematical model is simulated using Matlab Simulink programming language as indicated in figure 5 . 


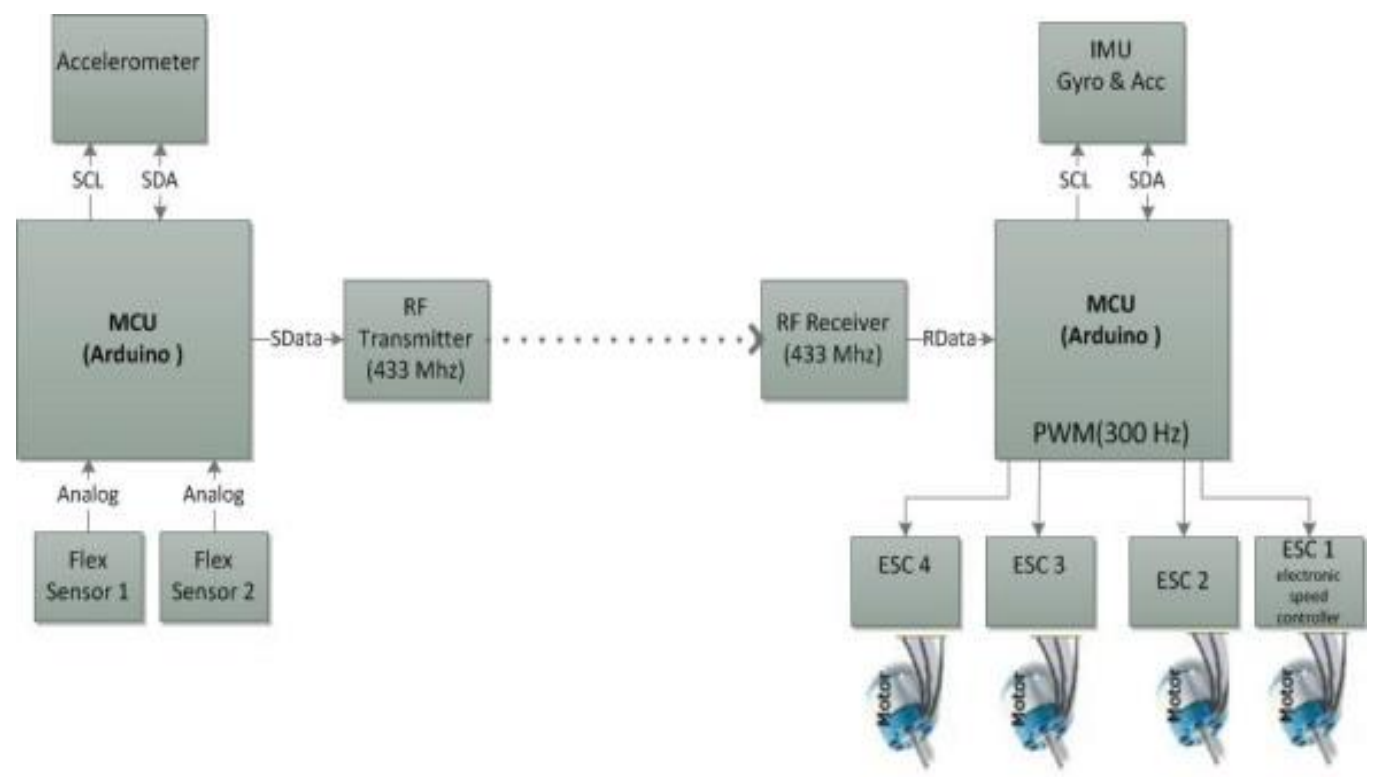

Fig 4 Hardware Implementation

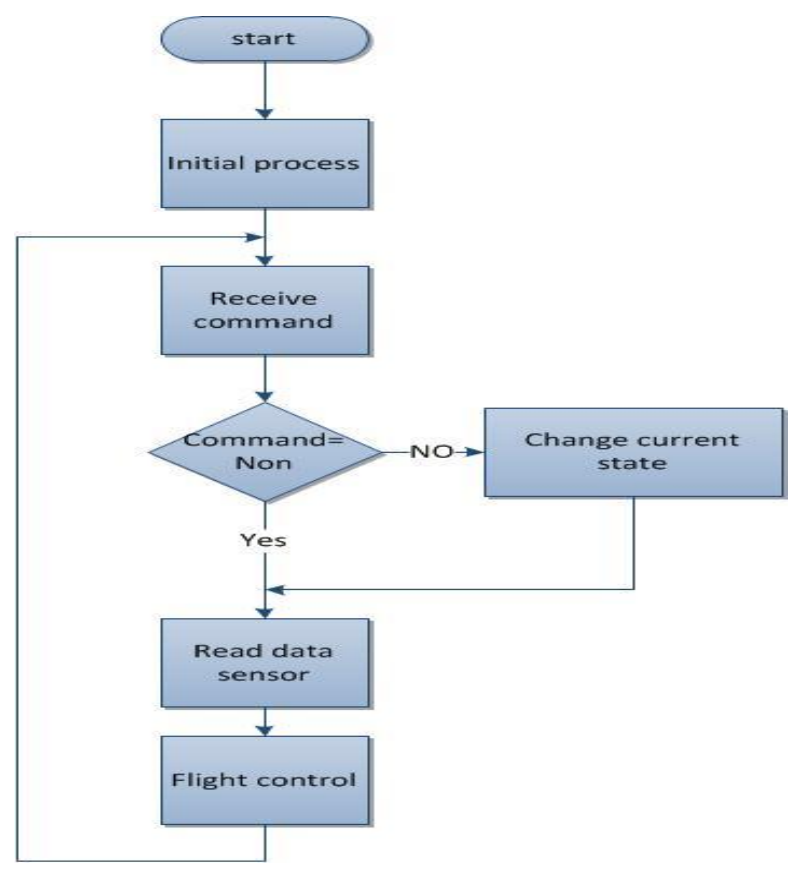

Fig. 5 Software Implementation

The quad copter system has two cases:

\section{Case 1:6-state ideal case}

The behavior of the system in ideal case (Neglecting sensors and motors effect) with LQR controller is designed to control only 6 of the 12 states available from the system, the angular velocities and the Euler angles. Fig. 7 shows the system response with pitch motion, fig. 8 shows the system response with roll motion and fig. 9 shows the system response with yaw motion. The state vector is $x=\left[\begin{array}{llllll}\phi & \theta & \psi & p & q & r\end{array}\right]^{T}$ and the motors angular velocities are $\omega_{1}, \omega_{2}, \omega_{3}, \omega_{4}$.It clear that change in motors angular velocities is step according to each motion. 


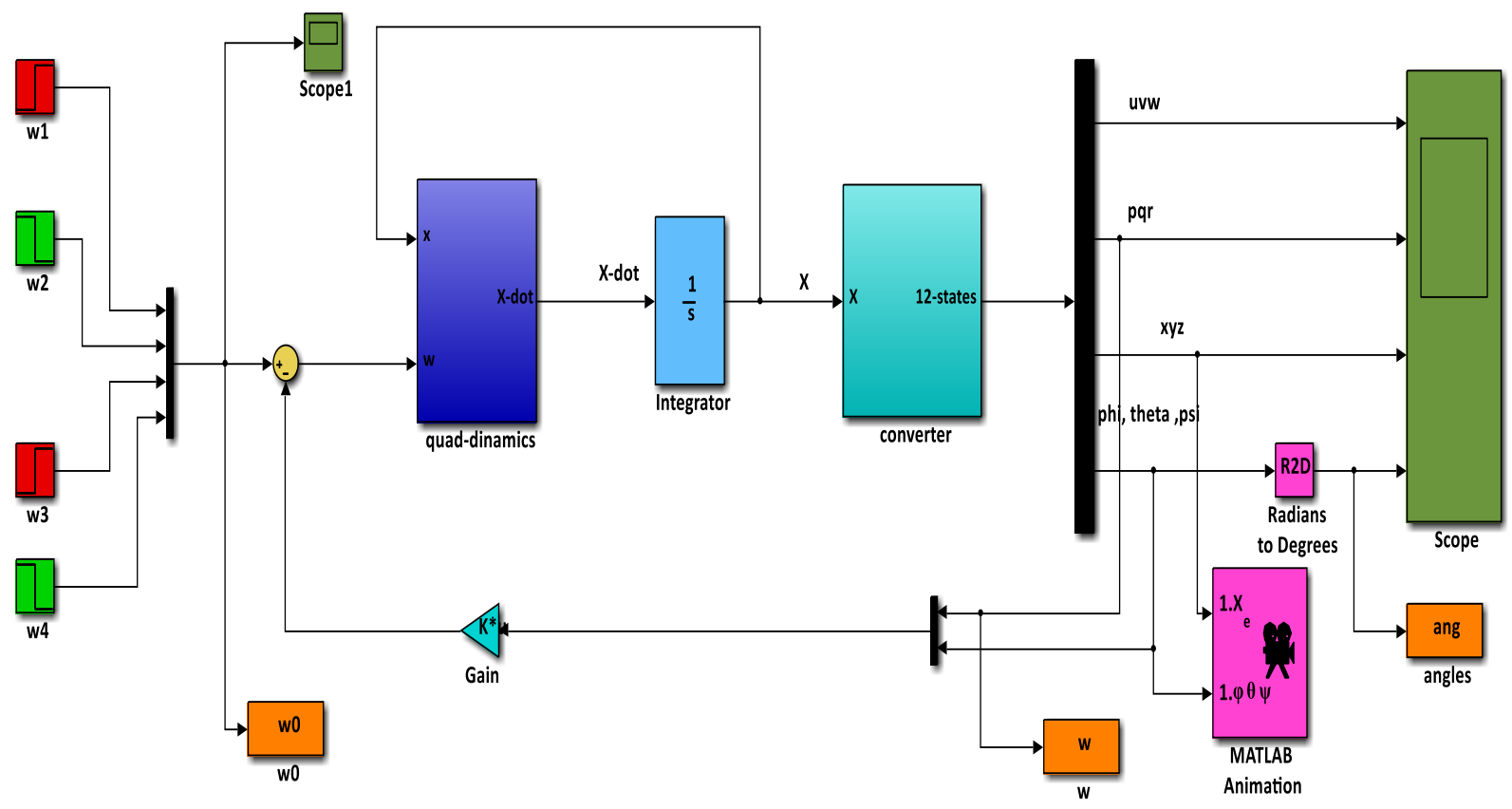

Fig.6 quad copter 6-state ideal case modeling
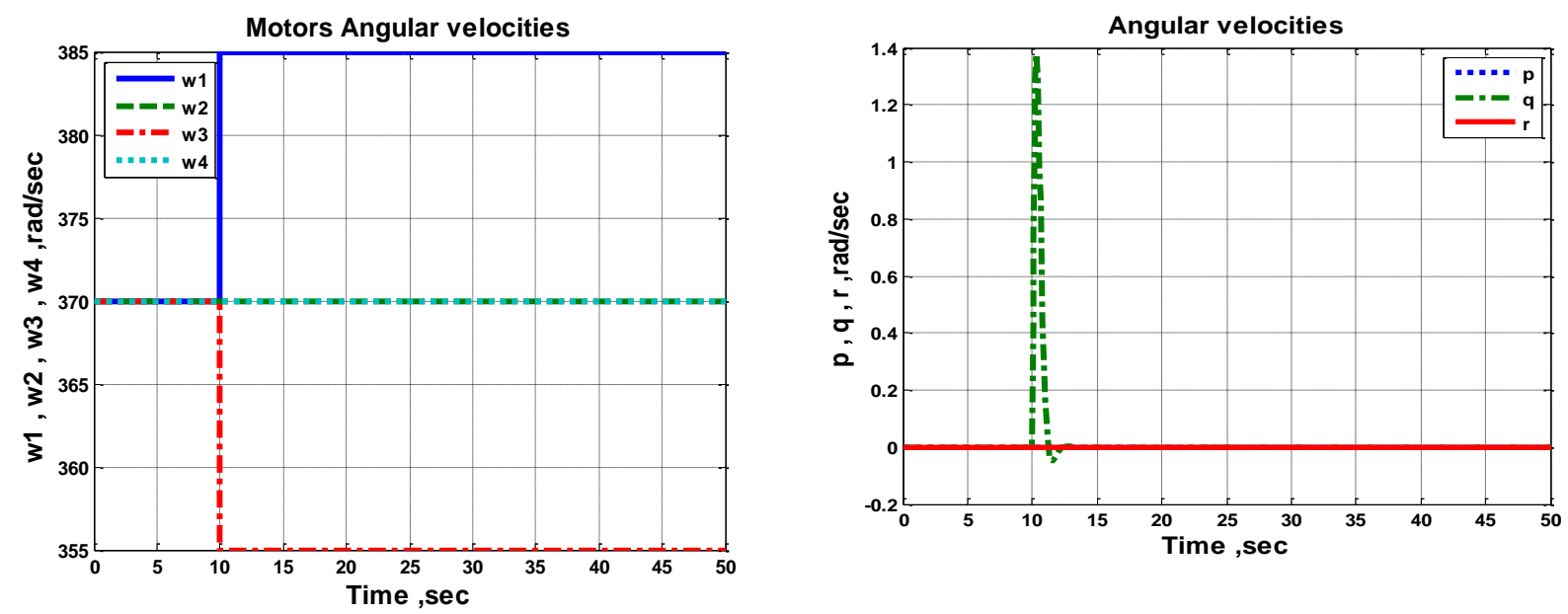

Fig.7 quad copter ideal case pitch motion
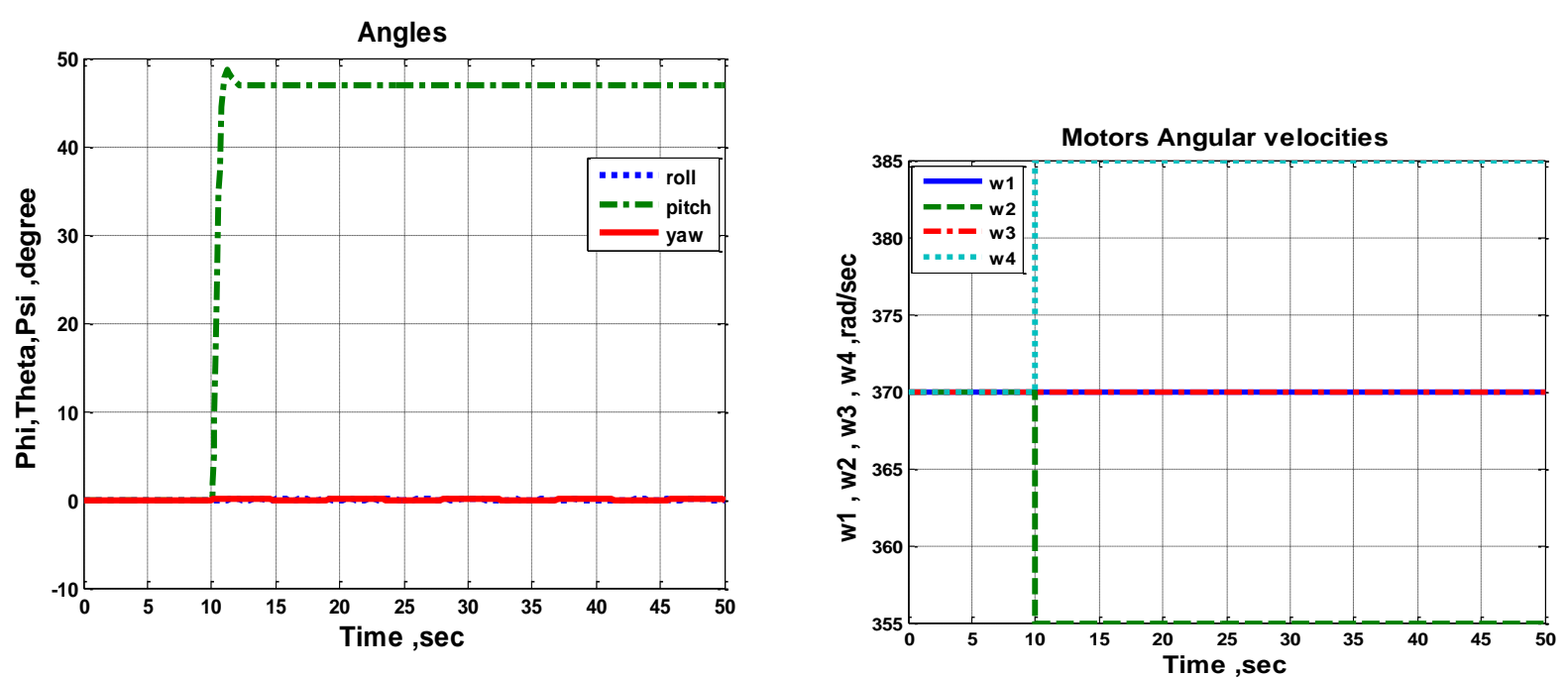

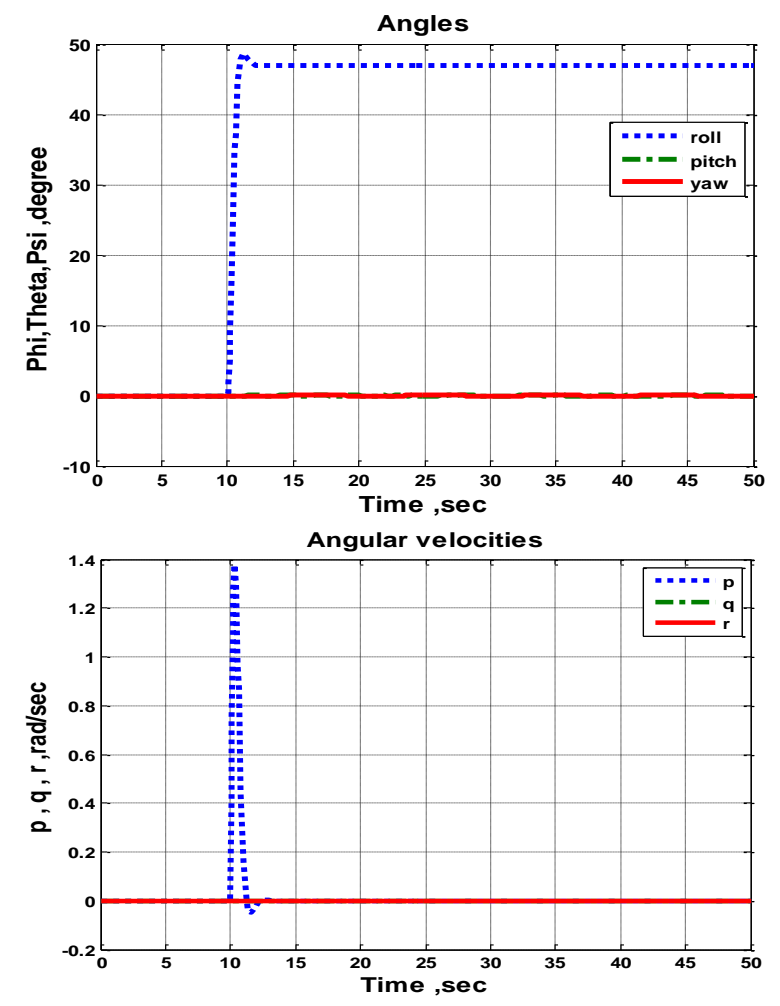

Fig.8 quad copter ideal case rolls motion
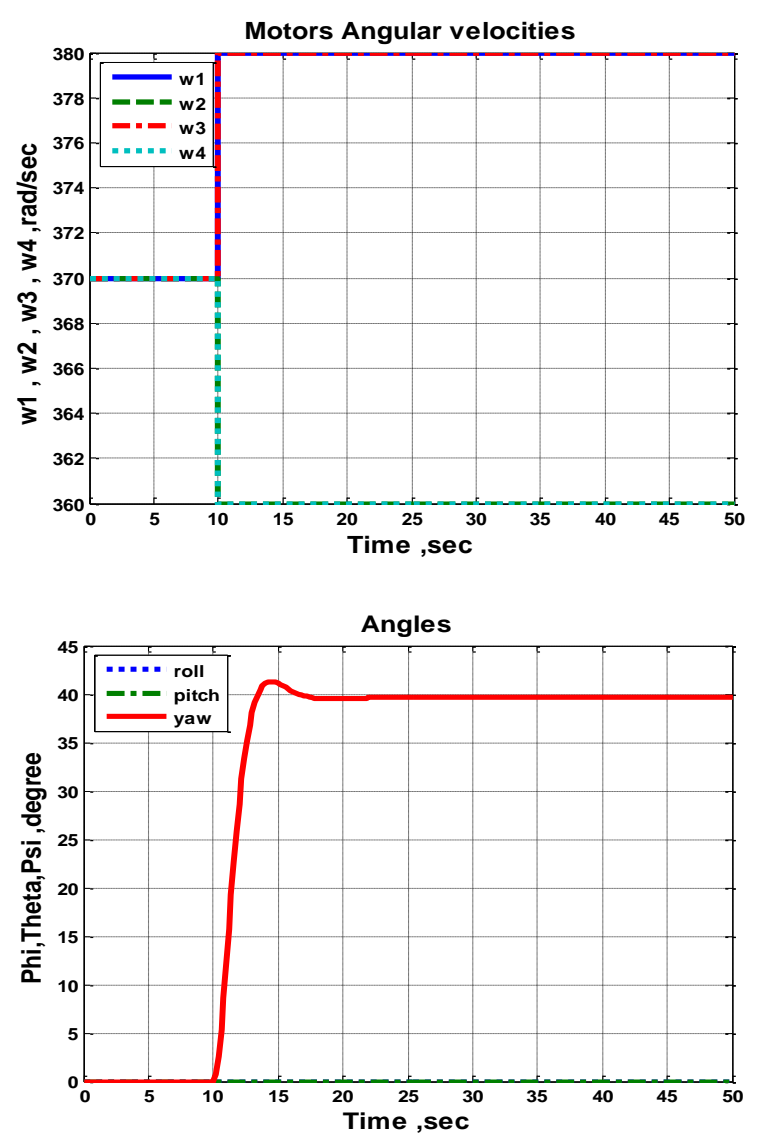

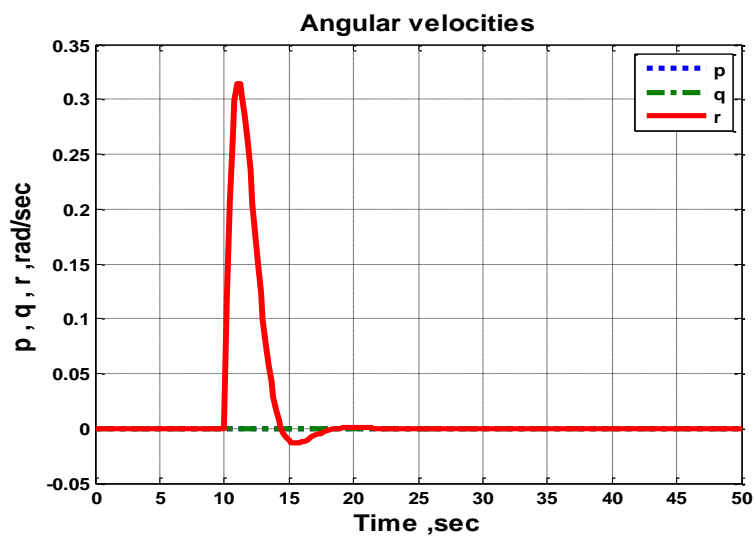

Fig.9 quad copter ideal case yaw motion

Pitch and roll motion curves have fast response for step variation (less than $3 \mathrm{sec}$ to reach stability) as only two having a change in their angular velocities, but for yaw motion it takes a quit more time to be stable (more than $22 \mathrm{sec}$ ) as all four motors angular velocities changed.

\section{Case 2: 6-state LQR controller practical case}

The effect of including motors dynamic and sensors in the control loop of a quad copter under LQR technique with kalman filter is illustrated in figure 10. Then, it will check if the LQR controller continues to maintain the quad copter leveled with only 6 states. The change in motors angular velocities is step according to each motion. Fig. 11 shows the system response with pitch motion, fig. 12 shows the system response with roll motion and fig.13 shows the system response with yaw motion. 


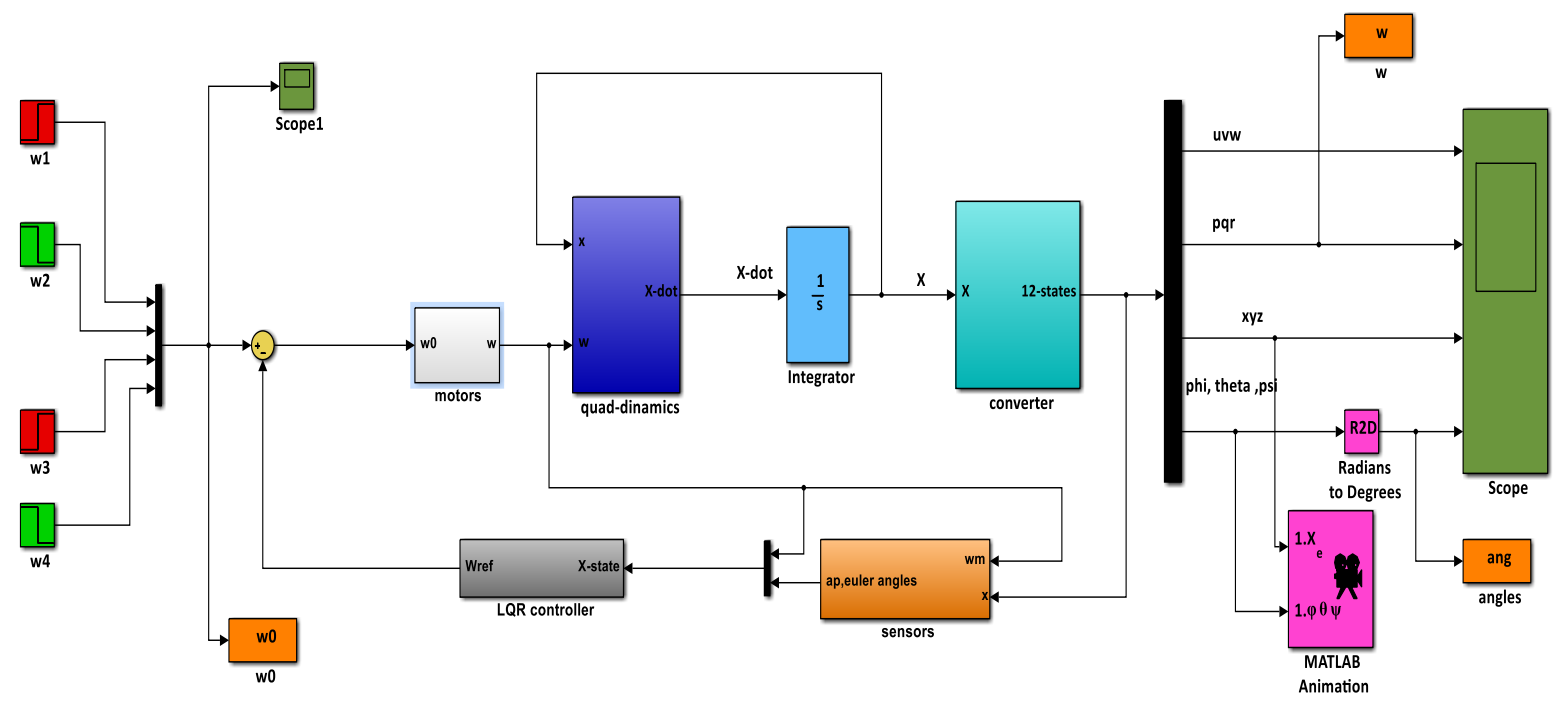

Figure 10 quad copter practical case modeling
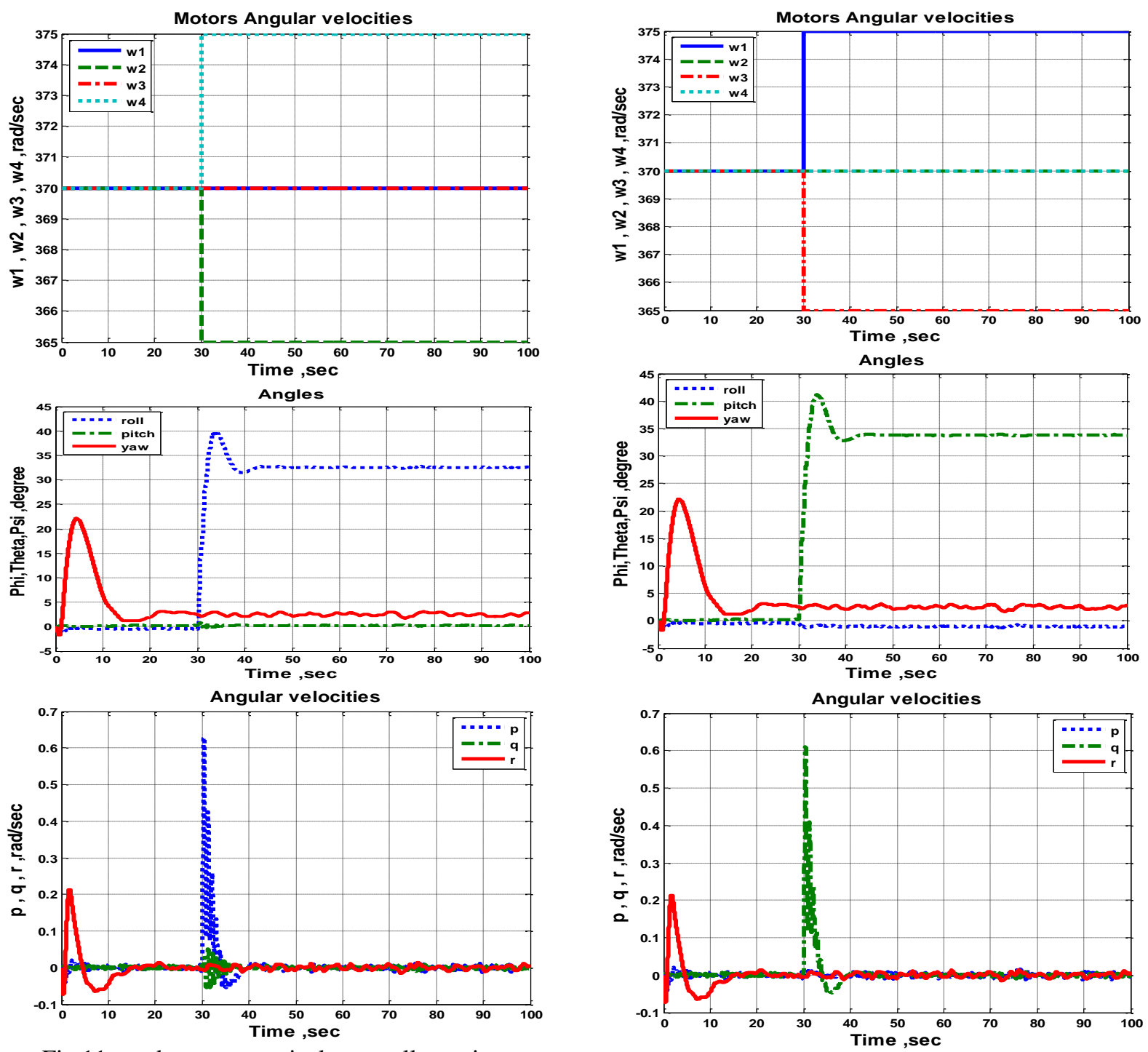

Fig.11 quad copter practical case rolls motion

Fig.12 quad copter practical case pitch motion 

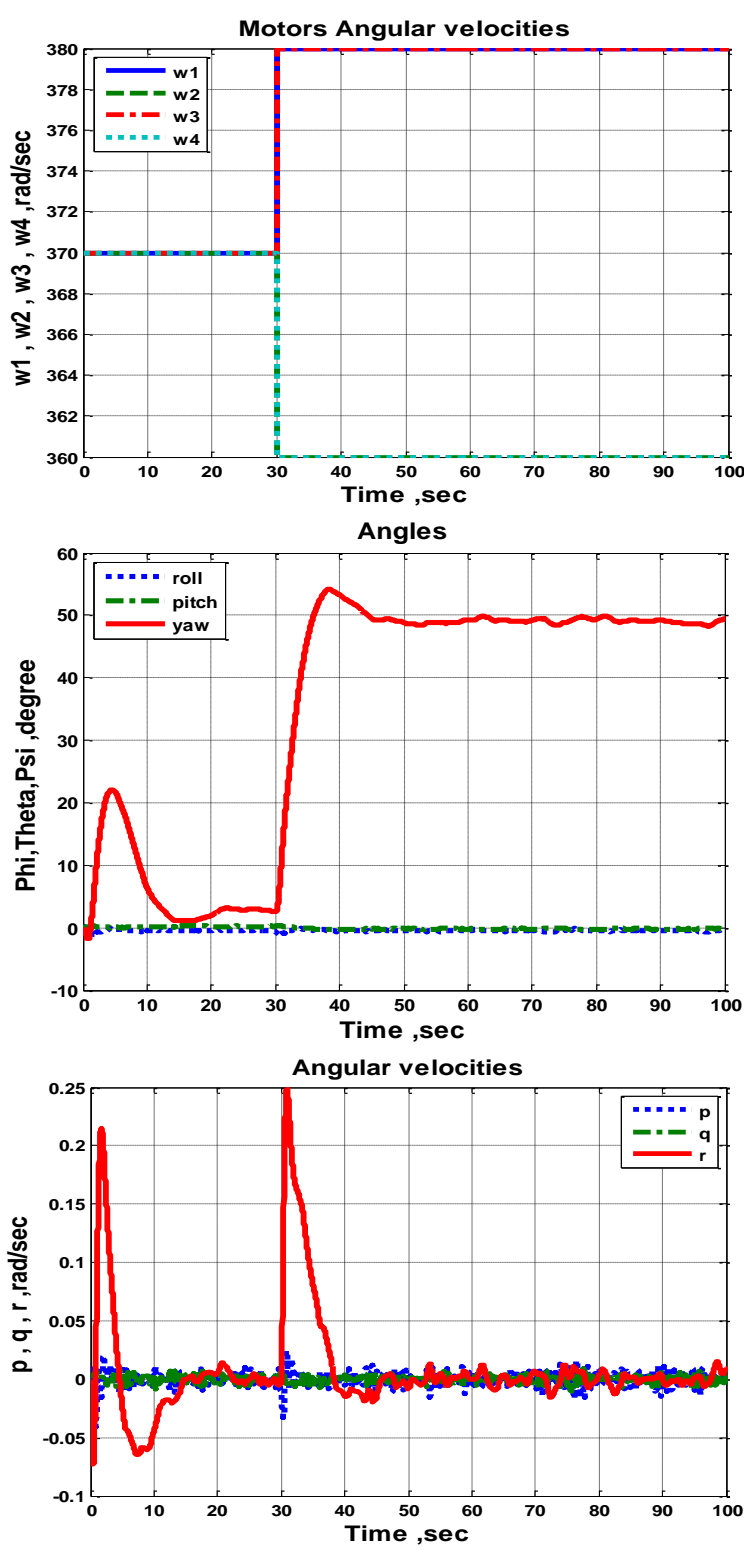

Fig 13 quad copter practical case yaw motion

The fluctuation shown is due to sensors reading noise. The appearance of small angels in roll and pitch motions beside the angle of variation is due to that all motors are not typical. Roll and pitch motion response for step variation is less than $12 \mathrm{sec}$ to reach stability, but for yaw motion it takes a quit more time to be stable (more than $20 \mathrm{sec}$ )

\section{CONCLUSIONS}

This paper presented the simulation and the implementation of a quad copter robot prototype, having a tri-axis accelerometer and a compass as its sensors. For a perfect analysis, the modeling of the aircraft's dynamics and kinematics for a computer simulation environment are preceded. A quad copter simulation model includes major aero dynamical effects modeled with blade element and momentum theory. In addition, the actuator's model was identified and all sensor delays and noises were taken into account. Real experiments were conducted with the same control parameters tuned in simulation. LQR controller used is sufficient and suitable for controlling the altitude and the attitude or Euler angles of the quad copter. 


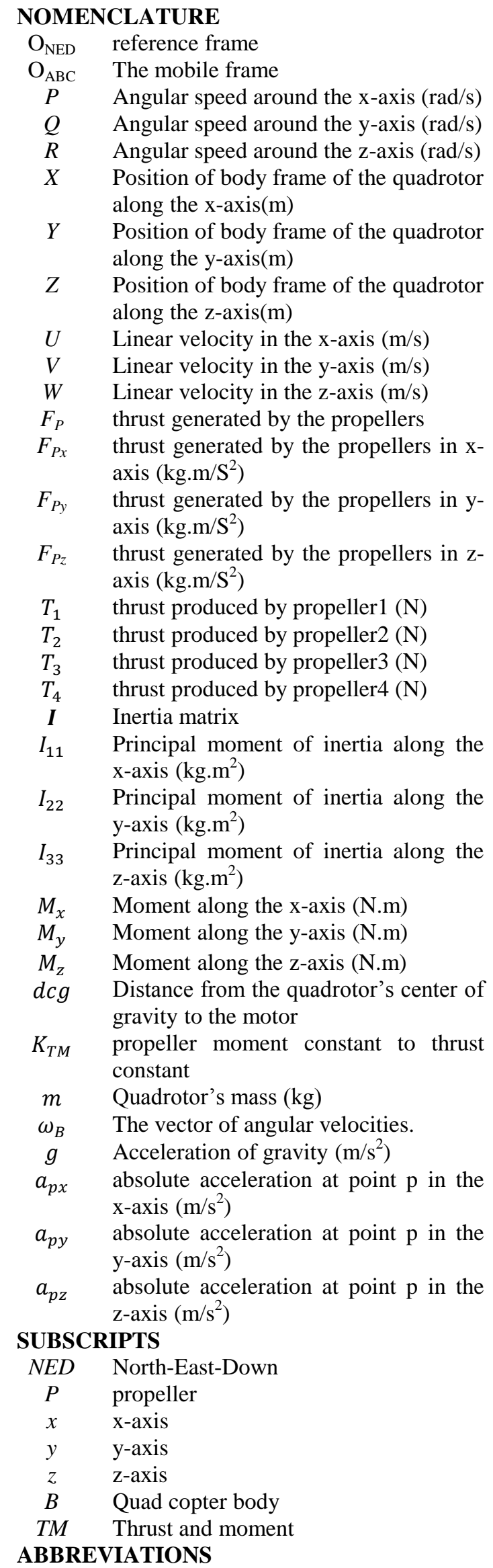

LQR Linear Quadratic Regulator

UAVs Unmanned Aerial Vehicles

$N \quad$ North

COG Center of Gravity

\section{REFERENCES}

1. K. P. Valavanis, "Advances in Unmanned Aerial Vehicles" vol. 33. Florida: Springer, 2007.

2. V. Martínez, "Modelling of the Flight Dynamics of a Quadrotor Helicopter," in Aerospace Sciences. Vol. Master of Science: Cranfield, 2007.

3. J.T.K. Ping, A. E. Ling, T. J. Quan, and C. Y. Dat. Generic "Unmanned Aerial Vehicle (UAV) for Civilian Application-A Feasibility Assessment and Market Survey on Civilian Application for Aerial Imaging". In 2012 IEEE Conf. on Sustainable Utilization and Development in Engineering and Technology, pages 289 -294, Oct. 2012.

4. B. Zhu and W. Huo. "Trajectory Linearization Control for a Quadrotor Helicopter". In 8th IEEE Int. Conf. on Control and Automation (ICCA), 2010, pages $34-39$, June 2010.

5. D. Mellinger and V. Kumar. "Minimum Snap Trajectory Generation and Control for Quadrotors". In IEEE Int. Conf. on Robotics and Automation (ICRA), 2011, pages 2520 -2525, may 2011.

6. G. V. Raffo, M. G. Ortega, and F. R. Rubio, "An Integral Predictive/Nonlinear H Infinity Control Structure for a Quadrotor Helicopter," Automatica, vol. 46, pp. 29-39, 2009.

7. Jean Walrand. "Kalman filter: Convergence". Lecture Notes, U. C. Berkeley, August 2009.

8. B. D. O. Anderson and J. B. Moore. "Optimal Control: Linear Quadratic Methods". PrenticeHall, Englewood Cliffs, New Jersey, 1990.

9. L. M. Argentim, W. C. Rezende, P. E. Santos, and R. A. Aguiar, "PID, LQR and LQR-PID on a Quadcopter Platform," in Proc. 2013 International Conference on Informatics, Electronics \& Vision, 2013,

10 Hafiz Farhaj Khan, Mansoor Saleem, Danish Mushtaq, and Faran Awais Butt, "A Comparative Analysis of Algorithms for Controlling the Attitude of an Unmanned Aerial Vehicle" Journal of Automation and Control Engineering Vol. 4, No. 3, June 2016 